\title{
The impact of expanding the screening interval for diabetic retinopathy in primary care centers
}

\author{
Sultan Fahad Magliah ${ }^{1}$, Wedad Mahmoud Bardisi ${ }^{2 *}$ and Maha Abdulaziz Alatta ${ }^{3}$ \\ ${ }^{1}$ Medical Doctor, King Saud University of Health Sciences, Medical College, Jeddah, Saudi Arabia \\ ${ }^{2}$ Consultant Family Physician, Ministry of Health General Directorate of Health Affairs Jeddah, Prince Abudulmajeed Health Center; Trainer in the Joint Family \\ Program of Family and Community Medicine Department, Jeddah, Saudi Arabia \\ ${ }^{3}$ Consultant Family Physician, Ministry of Health, Director, The Joint Program of Family and Community and Medicine Department, Jeddah, Saudi Arabia
}

\begin{abstract}
Objectives: This study aimed to estimate the prevalence of diabetic retinopathy (DR) and its determinant risk factors in people with diabetes attending primary care centers and to determine the impact of expanding the screening interval on the incidence and progression of (DR).

Methodology: This study was a cross-sectional chart review, that was conducted in three randomly selected primary care centers. A total of 250 diabetic patients had three consecutive annual screenings for DR from April 2014 to April 2017. At the initial visit, the ophthalmological findings were recorded. For three successive yearly screening, the screening results were assessed to estimate the changes that occurred in the prevalence, incidence, and progression of diabetic retinopathy in addition to the degree of association with the most predictable risk factors.

Results: The initial prevalence of DR was $15.2 \%$. Over the three-consecutive annual screening, the DR prevalence increased to $19.6 \%$, $22.4 \%$, and $25.6 \%$, respectively. Most cases (72\%) of DR were of the mild non-proliferative diabetic retinopathy (NPDR) type. The annual incidence of DR was $4.4 \%$, $2.8 \%$, and $3.2 \%$. The progression of DR occurred in $2(0.08 \%)$ cases at the second screening interval and in $6(2.4 \%)$ cases at the third screening interval. These changes were strongly associated with age, the duration of diabetes, HBA1C levels, hypertension, and insulin therapy (P-value 0.005). However, changes in the incidence and progression of DR over the three intervals were statistically non-significant (P-value 0.086).
\end{abstract}

Conclusion: DR, a serious microvascular complication of diabetes mellitus, is an asymptomatic disease with a slow onset and gradual progression. DR requires regular screening for early detection. The screening interval can be extended up to every three years in the very low-risk groups without threatening vision.

\section{Background}

Diabetes mellitus is one of the most prevalent diseases worldwide [1]. The Kingdom of Saudi Arabia has a high prevalence of diabetes mellitus at $23.7 \%$ [2], and this rate is expected to rise to $44.1 \%$ in 2022 [3]. DM is a well-known cause of microvascular and macrovascular complications. Diabetic patients often develop eye diseases as a complication. However, the most common and potentially sightthreatening of these complications is diabetic retinopathy (DR) [4]. The onset and progression of diabetic retinopathy is slow and gradual, advancing from mild non-proliferative diabetic retinopathy (NPDR), indicated by the presence of at least one microaneurysm, to moderate NPDR, indicated by the presence of hemorrhages, microaneurysms, and hard exudates. The severe form is characterized by hemorrhages and microaneurysms in four quadrants, with venous beading in at least two quadrants and intraretinal microvascular abnormalities in at least one quadrant. Proliferative diabetic retinopathy (PDR) is characterized by neovascularization, preretinal hemorrhages, hemorrhage into the vitreous, traction retinal detachments or macular edema (ME) [5]. Diabetic retinopathy is considered a frequent and leading cause of blindness, especially among the most productive age group between 20 and 60 years of age, with approximate estimates for the prevalence of retinopathy and vision-threatening retinopathy at $40.3 \%$ and $8.2 \%$, respectively (6). Of the 37 million blind people reported worldwide in the year 2002, diabetic retinopathy was responsible for 1.8 million (4.8\%) cases [6]. Diabetic retinopathy is considered the leading cause of blindness in the Kingdom of Saudi Arabia as well [7]. There is a wide discrepancy among the reported prevalences of DR. The overall global prevalence of DR among patients with type 2 diabetes is $27.23 \%$, while the prevalence of DR among different ethnic groups varies widely from $20.8 \%$ among Asians to $46.7 \%$ among Caucasians [8]. Moreover, the data collected from the Saudi National Diabetes Registry indicates that the overall prevalence of diabetic retinopathy is $19.7 \%$ (9). The risk factors are mainly related to age, the duration of diabetes and glycemic control; other factors, such as obesity, dyslipidemia and nephropathy, were variably associated $[9,10]$. In Saudi Arabia, nephropathy, neuropathy, insulin use, poor glycemic control, hypertension and male gender significantly increased the risk for diabetic retinopathy, while smoking, hyperlipidemia and obesity significantly were associated with reduced risk for diabetic retinopathy among Saudi type 2 diabetics [9]. Regular screening is the best method for the early detection of diabetic retinopathy and is strongly recommended since early detection has

*Correspondence to: Wedad Mahmoud Bardisi, Consultant Family Physician, Ministry of Health General Directorate of Health Affairs Jeddah, Prince Abudulmajeed Health Center; Trainer in the Joint Family Program of Family and Community Medicine Department, P.O. Box 35506 Jeddah 21849,Saudi Arabia, Tel: +966504528223; E-mail: wbardisi@yahoo.com

Key words: diabetic retinopathy, prevalence, risk factors, screening interval

Received: April 06, 2018; Accepted: April 18, 2018; Published: April 21, 2018 
the best chance of preventing retinal complications and blindness $[5,7,11,12]$. In the primary care setting, patients should be referred by a healthcare professional to an ophthalmologist or an optometrist who should perform a comprehensive eye examination including a dilated fundoscopy. Such an eye exam should be conducted annually and at regular intervals thereafter as recommended by the eye care professional [13]. Retinal photography can enhance the efficiency of screening and reduce costs [14]. With the rapid increase in the prevalence of diabetes, the burden and cost of annual screening are rising with no evidence of significant rewards in delaying the onset of DR, especially in newly diagnosed diabetics with controlled risk factors [15]. In diabetic patients with no retinopathy, there is reported evidence that biennial screening is cost-effective [14]. Screened patients who had no or nonproliferative retinopathy were found to have a very low risk of eventual blindness from diabetes [16].

In this study, we aimed to estimate the prevalence of diabetic retinopathy and its risk factors in the primary care setting and to measure the frequency of referral to an ophthalmologist for screening and the impact of expanding the DR screening interval among diabetic patients attending primary care centers.

\section{Methods}

This study was a cross-sectional chart review study using patient medical records. Three governmental primary health care centers were randomly selected from twelve centers equipped with well-established diabetic care units located in the city of Jeddah, Saudi Arabia. The total number of registered diabetics was 896 from April 2014 to April 2017. Medical records were reviewed, and only the complete medical records containing all the variables to be studied were included in this study. These variables included the following: the sociodemographic data [age, gender, height, weight, body mass index (BMI), smoking status]; systolic and diastolic blood pressure measurements obtained by the unit nurse for each visit or noted as a hypertensive patient undergoing treatment; type, onset and duration of DM; presence or absence of microvascular complications, such as retinopathy, neuropathy or nephropathy; presence or absence of macrovascular complications, such as stroke and cardiovascular disease (CVD); type of treatments prescribed (oral hypoglycemic, insulin combined with oral hypoglycemic or insulin alone); the frequency of ophthalmological referral; the results of the screening at the initial visit performed by ophthalmologists at the General Eye Hospital in Jeddah; the results of three consecutive screenings at regular one-, two- and three-year follow-up visits in the primary care center; and the measurement of hemoglobin Alc (HBA1C) and low-density lipoprotein (LDL) levels and the glomerular filtration rate (GFR). The level of HbA1C was measured using the method certified by the NGSP and standardized per the Diabetes Control and Complications Trial (DCCT) assay. The HBA1C level was categorized into three groups (less than 7, 7.1-10 and greater than 10). A value of less than 7 was considered controlled. The LDL level was used a marker for dyslipidemia, and a value greater than $100 \mathrm{mmol} / \mathrm{l}$ was considered high. The GFR was calculated using the National Kidney Foundation method of calculation, and a GFR less than 60 was considered to indicate nephropathy. The data were categorized as the dependent variable, which was diabetic retinopathy of any grade, and independent variables (age, sex, the duration of diabetes, BMI, HBA1C and LDL levels and the GFR). The screening intervals were categorized into four groups: the initial visit findings, and the findings after the first, second, and third annual screening intervals. The incidence of DR of any grade and the percentage of progression occurring over the three years were determined. This study was ethically approved by the
Directorate of Health Affairs, the Research and Studies Department, the Scientific and Biomedical committee, Jeddah, Saudi Arabia (ethical approval number H-02-J-002).

\section{Statistical analysis}

Statistical analysis was conducted using the Statistical Package for Social Sciences (SPSS) version 20.0 software (IBM Corp., Armonk, NY). A chi-square test (v2) was used for categorical variables, while the t-test was used for continuous variables. A P value of $<0.05$ was used as the level of significance. Independent-samples t-tests were used to evaluate the changes that occurred during the three screening intervals. Multinomial logistic regression analyses were performed to determine the most significant risk factors associated with the changes in DR. Adjusted ORs, $95 \%$ confidence intervals (CIs) and a P-value of $<0.05$ as the level of significance were used to assess the risk factors most closely related to changes in the incidence and progression of DR during the three years.

\section{Results}

A total of 250 medical records of those registered diabetic patients who were in primary care centers during the period from April 2014 to April 2017 were complete and fulfilled the inclusion criteria. There were $120(48.0 \%)$ males and $130(52.0 \%)$ females. The mean age \pm SD was $56 \pm 12$ years (min 23 -max 80 years). In the initial screening, there were 38 cases with retinopathy of any grade; these patients had a mean age \pm SD of $61 \pm 11$ years (95\% CI, 57.28-64.72\%), while the mean age of those who had no retinopathy was $54 \pm 10$ years $(95 \%$ CI, 52.27-55.73\%). This difference was statistically significant (P-value 0.001 ). There was an equal number of cases of DR among both genders, with 19 cases $(50.0 \%)$ for each, while other eye diseases (cataract and refractive errors) were present in $40(56.3 \%)$ females and $31(43.7 \%)$ males. Normal eye exam results were observed for 141 diabetics, 71 (50.4\%) females and 70 (49.6\%) males. Chi-square statistical tests were performed to estimate the degree of association between DR and the independent variables. There was no significant association with gender (P-value 0.668$)$, type of DM (P-value 0.080$)$, obesity for either DM1 or DM2 (P-value 0.980), or smoking (P-value 0.992). While the duration of DM, HBA1C level, uncontrolled diabetes, hypertension, dyslipidemia, nephropathy, and insulin treatment were all significantly associated (P-value $<0.005)$ (Table 1$)$.

Initially, the prevalence of DR of any grade was $15.2 \%$ (95\% CI 10.8-19.7\%). The other eye diseases (refractive errors and cataract) were present in 71 cases with a prevalence of $28.4 \%$ (95\% CI $22.8-34.2 \%$ ). The total number of DR cases increased to 49 cases at the first annual screening interval, with a prevalence of $19.6 \%$ (95\% CI 14-23.6\%). At the second screening interval at two years, there were 56 cases of DR and the prevalence was $22.4 \%$ (95\% CI 17.2-27.6\%). A total of 64 cases were detected in the third screening interval after three years with a prevalence of $25.6 \%$ (95\% CI 20.6-34.8\%) (Table 2).

The frequency and percentage of the different grades and diagnoses at the three screening intervals were also determined (Table 3 ).

The main changes that occurred during the three annual screening intervals were as follows:

204 (81.6.4\%) cases had no change from their primary initial diagnoses during the three screening intervals, while eleven cases (4.4\%) had a new onset at the first annual screening interval, 7 (2.8\%) cases had a new onset at the second interval, and $8(3.2 \%)$ cases had a new onset at the third interval. While 2 cases $(0.08 \%)$ progressed to the following grade of DR at the second screening interval, 6 cases 
Table 1. Association between socio-demographic characteristics and diabetic retinopathy and other eye disorders, $\mathrm{P}$ - value $<0.05=$ Significant association.

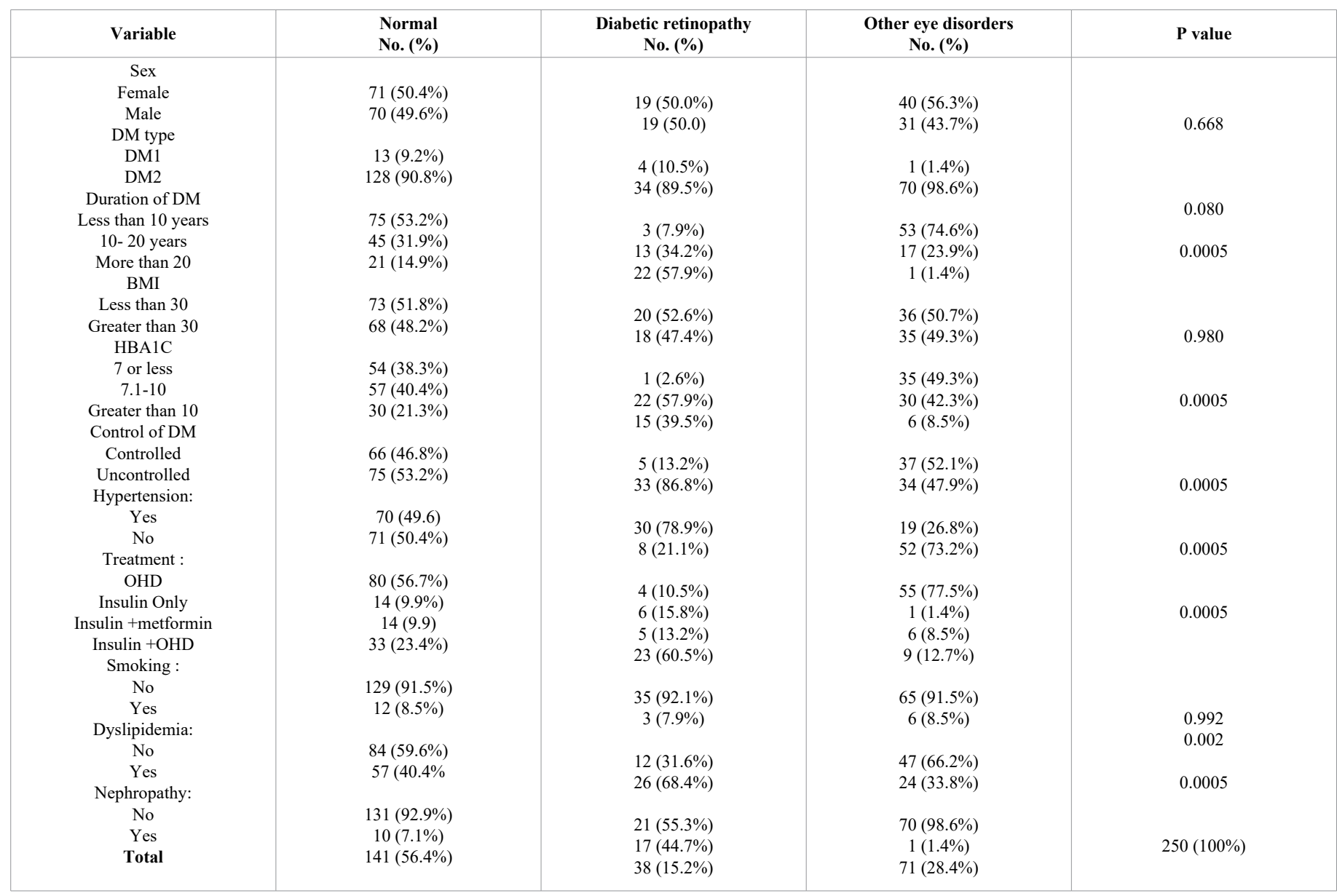

Table 2. The prevalence of diabetic retinopathy cases at each screening interval

\begin{tabular}{|c|c|c|c|}
\hline Screening Interval & Normal & Diabetic retinopathy & Other eye disease \\
\hline Initial & 141 & $38(15.2 \%)$ & $71(28.4)$ \\
\hline One year & 126 & $49(19.6 \%)$ & $75(30.0 \%)$ \\
\hline Two years & 116 & $56(22.4 \%)$ & 250 \\
\hline Three years & 103 & $64(25.6 \%)$ & 250 \\
\hline
\end{tabular}

Table 3. The frequency and percentage of the different diagnoses at the three screening intervals

\begin{tabular}{|c|c|c|c|c|}
\hline & \multirow[t]{2}{*}{ Initial finding } & \multicolumn{3}{|c|}{ Screening Interval } \\
\hline Diagnosis & & One year & Two years & Three years \\
\hline Normal & $141(56.4 \%)$ & $126(50.4 \%)$ & $116(46.4 \%)$ & $103(41.2 \%)$ \\
\hline Mild NPDR & $24(9.6 \%)$ & $35(14 \%)$ & $40(16.0 \%)$ & $42(16.8 \%)$ \\
\hline Moderate NPDR & $10(4.0 \%)$ & $10(4.0 \%)$ & $12(4.8 \%)$ & $14(5.6 \%)$ \\
\hline Severe NPDR & 0 & 0 & 0 & $2(0.8 \%)$ \\
\hline Maculopathy & $4(1.6 \%)$ & $4(1.6 \%)$ & $4(1.6 \%)$ & $6(2.4 \%)$ \\
\hline Cataract & $3(1.2 \%)$ & $4(1.6 \%)$ & $7(2 . \%)$ & $12(4.8 \%)$ \\
\hline Refractive errors & $68(27.2 \%)$ & $71(28.4 \%)$ & $71(28.4 \%)$ & $71(28.4 \%)$ \\
\hline
\end{tabular}

(2.4\%) progressed to another grade at the third screening interval. These changes over the three intervals were statistically non-significant (P-value 0.086).

A multinominal logistic regression with the calculation of the adjusted OR and 95\% CI revealed a significant association of these changes with age $>60$ years, a duration of diabetes of $>10$ years, an HBA1C level $>7.1$, hypertension, and insulin therapy, and these factors were strong predictors of the incidence and progression of DR (Table 4).

\section{Discussion}

Diabetic retinopathy is one of the main microvascular complications of diabetes mellitus and can threaten the sight and progress to blindness $[17,18] \cdot$ National and international studies that have been conducted to determine the prevalence of diabetic retinopathy have reported values that range from $15 \%$ in some studies to $36 \%$ in others [19-23]. Studies from Saudi Arabia's southern region reported a prevalence of $36.4 \%$, which is much higher than the previously reported prevalence of 
Table 4. Logistic regression analysis for the risk factors that might be associated with the main changes. aOR: adjusted odds ratio.

\begin{tabular}{|c|c|c|c|}
\hline Variable & aOR & $95 \%$ CI & P value \\
\hline $\begin{array}{l}\text { Duration of diabetes } \\
\quad \leq 10 \text { years } \\
>10 \text { years }\end{array}$ & 2.37 & $1.25-4.94$ & 0.001 \\
\hline $\begin{aligned} & \text { Age } \\
\leq & 60 \text { years } \\
> & 60 \text { years }\end{aligned}$ & 0.56 & $0.21-0.90$ & 0.002 \\
\hline $\begin{array}{l}\text { HBA } 1 \mathrm{C} \\
\quad<8 \\
>8\end{array}$ & 2.75 & $1.61-4.71$ & 0.001 \\
\hline $\begin{array}{l}\text { Hypertension } \\
\text { Yes } \\
\text { No }\end{array}$ & 0.67 & $0.43-0.89$ & 0.005 \\
\hline $\begin{array}{c}\text { Insulin use } \\
-\mathrm{NO} \\
\text {-Only or }+\mathrm{OHG}\end{array}$ & 2.04 & $1.67-4.56$ & 0.002 \\
\hline
\end{tabular}

$11.3 \%$ in the same region by Al-Khaldi, et al [24]. The last study using the Saudi National Diabetes Registry (SNDR) found that the overall prevalence of diabetic retinopathy of only 19.7\% [9]. In most of these studies, the risk factors were related to age, the duration of diabetes mellitus and poor glycemic control, while other factors, such as gender, obesity, smoking, nephropathy, and neuropathy, had no clear associations with diabetic retinopathy [9,25-29]. In this study the initial prevalence of diabetic retinopathy was $15.2 \%$ (95\% CI, 14.6-19.6\%). There was a significant association between diabetic retinopathy and the age of the patient. There was no significant association with gender, in contrast to some studies which reported a predominance for males $[28,30]$. There was no significant association with the type of diabetes mellitus, although studies around the world found that the prevalence of diabetic retinopathy was higher in people with type 1 diabetes than in those with type 2 diabetes [8,31,32]. Regarding association of obesity with diabetic retinopathy, this study found no significant association, since the relative risk (RR) of BMI (less than 30/more than 30) was 0.615 (95\% CI 0.334-1.130). Although a positive correlation of obesity with DR has been found in some studies [3,9,24,32-34], it was found to be protective in another studies [9)]. Controlling blood sugar is a protective factor $[5,8,11,32,33,35]$, and this association was proven in this study. Glycemic level was controlled in only $2.6 \%$ of the DR cases, while $97.4 \%$ of the DR cases were uncontrolled and had HBA1C level $>8.1$ (P-value 0.005); the relative risk was 0.25 (95\% CI 0.113-0.586). There was a strong association between insulin therapy and DR in this study. Similar findings have been reported in studies from China, Finland, Jordan and Saudi Arabia [9,23,24,28,36]. Hypertension has a strong association with DR (P-value 0.0001), and this was well documented in most of the other studies [24,32,33,37,38]. Like other studies, smoking was not associated with DR (P-value 0.992) $[5,9,11,24,39]$. The relationship between dyslipidemia and nephropathy has been proven in this study and other studies in Saudi Arabia and worldwide [8,21,25-28,40].

The screening program for diabetic retinopathy in primary care centers follows the Saudi National Diabetic Guideline for Primary Care, which recommends an annual referral to ophthalmologists or optometrist to perform a comprehensive ophthalmological examination for the early detection of DR, and the findings and recommendations of these professionals are sent back to the referring center.-Diabetic retinopathy has a delayed onset and a slow, gradual progression over many years and can even regress when the disease is in an early mild form [41-43]. Therefore, expanding the screening interval is permissible with no additional risks, especially in those with no retinopathy or with mild NPDR within the first 10 years of diabetes duration, since the prevalence of retinopathy during the first 10 years of a diabetes diagnosis has been found to be low, and progression in these cases was rare [43]. Furthermore, a study performed to determine the diabetic retinopathy screening interval based on the time from no retinopathy to laser therapy concluded that the screening interval for diabetic patients could be extended to two or three years. Patients with no retinopathy or who have a non-proliferative form have a very low risk of eventual blindness from diabetes [16].

In this study, it was found that the initial prevalence of diabetic retinopathy was $15.2 \%$. Most of these cases had mild nonproliferative retinopathy. There was a low but definite annual incidence in these patients; however, progression was relatively infrequent and was associated with age, the duration of diabetes, hemoglobin A1c levels, hypertension, and insulin therapy.

In view of these findings, we concluded that regular screening for DR, and improving treatment for diabetics with new onset of retinopathy is of paramount importance to decrease the incidence and progression. Based on the findings from this study it is suggested that diabetic screening intervals could be increased to every 3 years in low risk patients.

Considering the increasing prevalence of diabetes mellitus that will overwhelm the diabetic care system, it would be more cost-effective to expand the screening interval for low risk groups. Applying a risk-based model of screening for DR can help in estimating the appropriate screening interval for DR [15]. Primary prevention is highly recommended to control the risk factors that will delay the onset and progression of diabetic retinopathy.

\section{Limitations}

Limitations of this study are the small sample size and the variation in the initial presentations and in the preregistration history of diabetes and its complications in the patients who were included in the study. This study was performed in the primary care setting, which is different from a hospital-based setting that may affect the outcome.

\section{Acknowledgments}

The authors would like to acknowledge the efforts of the data collector who worked hard to review the charts manually. We also appreciate the work of the statistician who conducted all the statistical analyses. We acknowledge all the diabetic unit teams who cooperated with the research team. 


\section{References}

1. Ogurtsova K, da Rocha Fernandes JD, Huang Y, Linnenkamp U, Guariguata L, et al (2017) IDF Diabetes Atlas: Global estimates for the prevalence of diabetes for 2015 and 2040. Diabetes Res Clin Pract 128: 40-50.

2. Mansour M Al-Nozha (2004) Diabetes mellitus in Saudi Arabia. Medical Services Department, Saudi Arabian Armed.

3. Al-Quwaidhi AJ, Pearce MS, Sobngwi E, Critchley JA, O'Flaherty M (2014) Comparison of type 2 diabetes prevalence estimates in Saudi Arabia from a validated Markov model against the International Diabetes Federation and other modelling studies. Diabetes Res Clin Pract 103: 496-503. [Crossref]

4. Cai X, McGinnis JF (2016) Diabetic Retinopathy: Animal Models, Therapies, and Perspectives. Journal of Diabetes Research. Hindawi 10: 1-9.

5. El-Bab MF, Shawky N, Al-Sisi A, Akhtar M (2012) Retinopathy and risk factors in diabetic patients from Al-Madinah Al-Munawarah in the Kingdom of Saudi Arabia. Clinical Ophthalmology, Auckland, NZ 6: 269-276

6. JH K, BJ O, MC L, SM H, R K, et al. (2004) The prevalence of diabetic retinopathy among adults in the United States. Arch Ophthalmol 122: 552-563.

7. Al-Turki YA (2002) Blood sugar control, ophthalmology referral and creatinine level among adult diabetic patients in primary health care, Riyadh, Saudi Arabia. Saudi Med J 23: $1332-1334$.

8. Yau J, Rogers SL, Kawasaki R (2012) Global prevalence and major risk factors of diabetic retinopathy. Diabetes.

9. Al-Rubeaan K, Abu El-Asrar AM, Youssef AM, Subhani SN, Ahmad NA, et al. (2015) Diabetic retinopathy and its risk factors in a society with a type 2 diabetes epidemic: a Saudi National Diabetes Registry-based study. Acta Ophthalmol 93: e140-e147.

10. Elwali ES, Almobarak AO, Hassan MA, Mahmooud AA, Awadalla H, Ahmed MH. Frequency of diabetic retinopathy and associated risk factors in Khartoum, Sudan population based study. Int J Ophthalmol 10: 948-954.

11. Al-Maskari F, El-Sadig M (2007) Prevalence of diabetic retinopathy in the United Arab Emirates: a cross-sectional survey. BMC Ophthalmology 1: 11.

12. Aiello LP (2014) Diabetic Retinopathy and Other Ocular Findings in the Diabetes Control and Complications Trial/Epidemiology of Diabetes Interventions and Complications Study. Diabetes Care American Diabetes Association 37: 17-23.

13. Looker HC, Nyangoma SO, Cromie DT, Olson JA, Leese GP, et al. (2013) Predicted impact of extending the screening interval for diabetic retinopathy: the Scottish Diabetic Retinopathy Screening programme. Diabetologia. Springer Berlin Heidelberg 56: $1716-1725$.

14. Chalk D, Pitt M, Vaidya B, Stein K (2012) Can the Retinal Screening Interval Be Safely Increased to 2 Years for Type 2 Diabetic Patients Without Retinopathy? Diabetes Care. American Diabetes Association 35: 1663-1668.

15. Chatziralli I, Sergentanis TN, Crosby-Nwaobi R, Winkley K, Eleftheriadis H, et al. (2017) Model for Risk-Based Screening of Diabetic Retinopathy in People With NewlyDiagnosed Type 2 Diabetes Mellitus. Invest Ophthalmol Vis Sci. The Association for Research in Vision and Ophthalmology 58: BIO99-BIO105.

16. Hughes D, Nair S, Harvey JN (2017) Determining diabetic retinopathy screening interval based on time from no retinopathy to laser therapy. J Med Screen 2: 170-175.

17. Jeffrey R. Willis (2017) Vision-Related Functional Burden of Diabetic Retinopathy Across Severity Levels in the United States 135: 926-932.

18. Claudia RLC (2017) Predictors of Development and Progression of Retinopathy in Patients with Type 2 Diabetes: Importance of Blood Pressure Parameters. Scientific Reports 7: 4867

19. Sunita M (2017) Prevalence of Diabetic Retinopathy in Urban Slums: The Aditya Jyot Diabetic Retinopathy in Urban Mumbai Slums Study-Report 2. Opthalmic Epidemology 24: 303-310.

20. Raman R (2017) Incidence and Progression of Diabetic Retinopathy in Urban India: Sankara Nethralaya-Diabetic Retinopathy Epidemiology and Molecular Genetics Study (SN-DREAMS II), Report 1. Opthalmic Epidemology 24: 294-302.

21. Sasongko MB, Widyaputri F, Agni AN, Wardhana FS, Kotha S, et al. (2017) Prevalence of Diabetic Retinopathy and Blindness in Indonesian Adults With Type 2 Diabetes. Am J Ophthalmol 181: 79-87. [Crossref]

22. Zhang G, Chen H, Chen W, Zhang M (2017) Prevalence and risk factors for diabetic retinopathy in China: a multi-hospital-based cross-sectional study. Br J Ophthalmol 101: $1591-1595$
23. Rabiu MM, Bdour A1 MD, Abu Ameerh MA, Jadoon MZ. Prevalence of blindness and diabetic retinopathy in northern Jordan. Eur J Ophthalmol 25: 320-327.

24. Ahmed RA, Khalil SN, Al-Qahtani MAA (2016) Diabetic retinopathy and the associated risk factors in diabetes type 2 patients in Abha, Saudi Arabia. J Family Community Med 23: 18-24.

25. El-Asrar AM, Al-Rubeaan KA, Al-Amro SA, Kangave D, Moharram OA (1998) Risk factors for diabetic retinopathy among Saudi diabetics. Int Ophthalmol 22: 155-161.

26. Al-Adsani AM (2007) Risk factors for diabetic retinopathy in Kuwaiti type 2 diabetic patients. Saudi Med J 28: 579-583.

27. Abougalambou SSI (2015) Risk factors associated with diabetic retinopathy among type 2 diabetes patients at teaching hospital in Malaysia. Diabetes \& Metabolic Syndrome: Clinical Research \& Reviews 9: 98-103.

28. Risk factors of diabetic retinopathy and sight-threatening diabetic retinopathy: a crosssectional study of 13473 patients with type 2 diabetes mellitus in mainland China. $B M J$ 7: $\mathrm{e} 016280$.

29. Kahtani ESA (2016) Assessment of the prevalence and risk factors of ophthalmoplegia among diabetic patients in a large national diabetes registry cohort. BMCOphthalmology 16: 118 .

30. Asrar El AA (1999) Risk Factors in Diabetic Retinopathy. Saudi journal of ophthalmology.

31. Minuto N (2012) Retinopathy screening in patients with type 1 diabetes diagnosed in young age using a non-mydriatic digital stereoscopic retinal imaging. Journal of Endocrinological Investigation 35: 389-394.

32. Ting DS, Cheung GC, Wong TY (2016) Diabetic retinopathy: global prevalence, major risk factors, screening practices and public health challenges: a review. Clin Exp Ophthalmol 44: 260-277.

33. Alaboud AF, Tourkmani AM, Alharbi TJ, Alobikan AH, Abdelhay O, et al. (2016) Microvascular and macrovascular complications of type 2 diabetic mellitus in Central, Kingdom of Saudi Arabia. Saudi Med J 37: 1408-1411.

34. Prevalence and determinants of diabetic retinopathy in $\mathrm{Al}$ hasa region of saudi arabia primary health care centre based cross-sectional survey, 2007-2009. Middle East Afr J Ophthalmol 17: 257-263

35. Laatikainen L, Ojamo M, Rudanko S-L, Summanen P, Keinänen-Kiukaanniemi S, et al. (2016) Improving visual prognosis of the diabetic patients during the past 30 years based on the data of the Finnish Register of Visual Impairment. Acta Ophthalmol 94: 226-231.

36. Naidoo K (2014) Prevalence and causes of vision loss in sub-Saharan Africa: 19902010. BMJ 98: 612-618.

37. Mera F. Haddad (2017) Public awareness of common eye diseases in Jordan. BioMed Central 17: 177.

38. Marahrens L (2017) Patients' preferences for involvement in the decision-making process for treating diabetic retinopathy. BioMed Central 17: 139.

39. Alrawahi AH, Rizvi SG, Al-Riyami D, Al-Anqoodi Z (2012) Prevalence and risk factors of diabetic nephropathy in omani type 2 diabetics in Al-dakhiliyah region. Oman Med J 27: 212-216. [Crossref]

40. Al-Bdour MD (2008) Risk Factors for Diabetic Retinopathy among Jordanian Diabetics. Medknow Publications 15: 77-80.

41. Gibson DM (2017) Frequency and predictors of missed visits to primary care and eye care providers for annually recommended diabetes preventive care services over a twoyear period among U.S. adults with diabetes. Prev Med 105: 257-264.

42. Konstantinidis L (2017) Awareness and practices regarding eye diseases among patients with diabetes: a cross sectional analysis of the CoDiab-VD cohort. BioMed Central 17: 56.

43. Voigt M, Schmidt S, Lehmann T, Köhler B, Kloos C, et al. (2017) Prevalence and Progression Rate of Diabetic Retinopathy in Type 2 Diabetes Patients in Correlation with the Duration of Diabetes. Exp Clin Endocrinol Diabetes.

Copyright: (C2018 Magliah SF. This is an open-access article distributed under the terms of the Creative Commons Attribution License, which permits unrestricted use, distribution, and reproduction in any medium, provided the original author and source are credited. 